\title{
DIE UNGARISCHEN TASCHENMESSERSPIELE (EINE TYPOLOGIE)
}

\author{
Imre KATONA \\ H-1084 Budapest, Német u. 23. III/2., Ungarn
}

Bis in die jüngste Vergangenheit war das zusammenklappbare Taschenmesser (ung. bicska) ein unverzichtbarer Gegenstand der gesamten männlichen Bevölkerung Ungarns. Die Geschicklichkeits-Taschenmesserspiele wurden von der Schulzeit bis ins Erwachsenenalter gespielt. Eine ihrer Bedeutung entsprechende umfassende Studie ist bisher weder über die Messerer/Messerschmiede noch über die Taschenmesserspiele erschienen. Vorliegende Arbeit möchte diesem Mangel zumindest in bezug auf die Taschenmesserspiele abhelfen und in Form einer Typologie eine Basis für weitere Forschungen legen.

Beiläufig haben sich die seit etwa 100 Jahren laufenden ungarischen Spielforschungen zwar mit den Taschenmesserspielen beschäftigt, doch waren die Beschreibungen in mehreren Fällen skizzenhaft und die Sammlungsarbeiten gebietsmäßig unausgewogen: Die Mehrheit der 38-42 Varianten (22) stammten aus der Großen Ungarischen Tiefebene, in die übrigen teilten sich die größeren Landschaften etwas gleichmäßiger: Kleines Tiefland (8), Transdanubien (5), Siebenbürgen (4) und Oberungarn (2). Die herausragende Rolle der im übrigen bei weitem nicht traditionellsten Tiefebene kann keinesfalls zufällig sein.

Die verschiedenen Beschreibungen erwähnen nicht immer die übliche Zeit der Spiele, im allgemeinen wurde von Frühling bis zum Herbst und innerhalb dieser Zeit während des Schuljahres und im Sommer gespielt; fallweise wurde gesondert auf das Weiden hingewiesen. Der Ort wird weit öfter erwähnt: Man spielte draußen im Freien, nur ausnahmsweise auf dem Schulhof oder in geschlossenen Räumen, wenn letzteres der Charakter des Spiels erlaubte. Man schätzte den nicht zu lockeren und nicht zu harten Boden, in dem das herabgeworfene Messer stecken blieb; nur als Ausnahme wird ein Sandkasten erwähnt. Die kleine Spielfläche wurde nötigenfalls gesäubert und geglättet. Manchmal wurde die ganze Oberfläche gestutzt: einander dicht kreuzende parallele Schnitte wurden gezogen. Die sorgfältig vorbereitete Spielfläche blieb eventuell längere Zeit in Gebrauch; dem jeweiligen Spieltyp gemäß wurde sie unterteilt.

Das Spielen mit dem für gefährlich gehaltenen Taschenmesser befürworteten im allgemeinen weder die Eltern noch die Erzieher, die strengeren verboten es sogar, obwohl keine Angaben über ernsthaftere Verletzungen bekannt sind. Auf jeden Fall lehrten nicht die Erwachsenen, sondern die größeren Jungen die kleineren. Die 


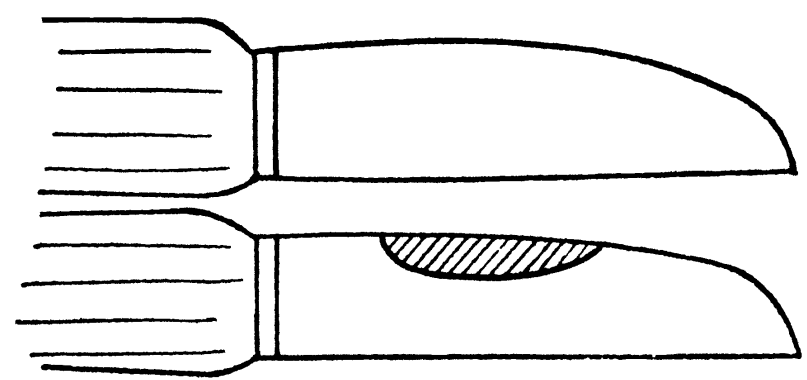

Abb. 1. Die beiden Seiten der Klinge: die glatte und die gekerbte Seite (BAKOS 1953: 262)

6-7jährigen lernten noch, die der Altersklasse der 8-14jährigen übten auch schon die schwierigsten Spiele, und im 15.-16. Lebensjahr hörten sie damit wieder auf. (Über Taschenmesserspiele Erwachsener gibt es nur vereinzelte Angaben.)

Jedes Taschenmesserspiel wurde gemeinsam gespielt, je nach Typ konnte man auch Paare bilden, durchschnittlich spielten aber 4-6 Teilnehmer, fallweise konnten an mit geregelt verringerter Zahl gespielten Varianten auch Gruppen von 8-10, 10-12 und ausnahmsweise 12-14 teilnehmen.

In der entsprechenden Gruppe der Kinderspiele des Volkes spielen die verwendeten Geräte nicht immer die Hauptrolle, doch bei den Taschenmesserspielen hängt fast jedes Moment mit dem Taschenmesser zusammen: Vorbereitung und Einteilung der Spielfläche, Auslosen der Reihenfolge der Spieler, Erfüllung verschiedenster Aufgaben, ja meist auch noch der Abschluss mit Belohnung oder Bestrafung und selbst die glatte und die zur Erleichterung des Aufklappens geriffelte Seite der Klinge spielen eine wichtige Rolle (Abb. 1). Die Anfänger mochten zumeist mit kleineren Kinder oder billigen Taschenfeiteln mit Holzgriff spielen, die Geübteren hatten ein Messer mit Horn- oder beinernem Griff. Jeder spielte mit seinem eigenen Messer, nur ausnahmsweise benutzten sie gemeinsame. Sonstige Hilfsmittel (z. B. Pflöcke, Kieselsteine, Erdklumpen, Tücher usw.) spielten eine völlig untergeordnete Rolle.

Zwar wurde die Volksterminologie der Taschenmesserspiele nicht immer aufgezeichnet, eventuell hatten die Gewährsleute sie selbst schon vergessen, doch kommen die Benennungen der einzelnen Spielvarianten (37) der Zahl der Varianten nahe, was eine auffällige Vielfalt bedeutet; vielleicht weist es auf die relativ neuzeitliche Entstehung dieser Typenfamilie und ihre Übernahme von anderen Völkern hin. Ein Teil dieser vielfältigen Benennungen (8) verweisen auf schwer interpretierbare spielerische Wortbildungen (z. B. ung. dani, dödölle usw.), kaum weniger (7) auf für typisch gehaltene Bewegungen (z. B. mit der Spanne messen, werfen, spannen, hinwerfen usw.), eine gleiche Zahl von Bezeichnungen (je 6) nennt das Gerät selbst (das Taschenmesser) bzw. die Phantasiegewinne (z. B. Land, Ackerfeld, Schloß, Pferd usw.), relativ oft kommen auf den Priester bezügliche Termini (priesterlich, Priesterei usw.) vor, die vielleicht noch Erinnerungen an die kirchenzehntpflichtige Leibeigenschaft sind, und schließlich konnten in einigen Fällen (3) die Spielvarianten auch nach geschickten, flinken Tieren (Katze, Grashüpfer) benannt werden. 
Die Taschenmesserspiele haben zwar Gesellschaftsspielcharakter, sind aber keine Mannschaftsspiele, weil nach vorher festgelegter Reihenfolge jeder mit seiner eigenen Leistung an diesen Geschicklichkeitswettbewerben teilnimmt. Die Einteilung der Spielfläche und die Reihenfolge der Würfe muß zwar vorweg bestimmt werden, doch erwähnen die Sammler nur in fünf Fällen die Auslosung, meist verweisen sie nur auf Parallelen bei anderen Spielen. So konnte z. B. der Anfangsspieler durch Abzählen oder durch Finden eines in der Faust versteckten Kiesels oder anderen Gegenstandes ausgelost werden, aber zum Charakter des Spiels paßte am besten das Herausfinden der glatten oder eingekerbten Klingenseite, ohne sie zu sehen oder vor dem Wurf. Bei einem Geländegewinn-Spieltyp kommt eine nicht eben geschmackvolle Auslosung vor: Am Spielfeldrand stehend, spuckte einer auf seinen Handrücken und schlug mit dem Zeigefinger darauf; auf wessen Feldteil der Speichel spritzte, der konnte das Spiel beginnen. Die Reihenfolge der Würfe ließ sich bei Kreisspielen leicht bestimmen: sie setzte sich im Uhrzeigersinn fort; für das Gegenteil gibt es nur eine einzige Angabe aus Transdanubien.

Über den Abschluß der Spieltypen, also die Belohnung der Sieger und Bestrafung der Verlierer bzw. Letzten, wissen wir etwas mehr als über die Auslosung des Beginnenden. Die Taschenmesserspiele sind typische Geschicklichkeitskämpfe, Wettbewerbe, dementsprechend gibt es immer Erste und Letzte; Belohnung und Bestrafung sind aber nicht unbedingt Bestandteile des Spielschlusses. Vielfach genügt der Ruhm, wenn jemand z. B. fehlerfrei oder als Erster die 6-12 verschiedenen, immer schwerer werdenden Würfe schafft, bei einem anderen Typ als Erster den Mittelpunkt des Kreises trifft, am weitesten weg von der festgelegten, gemeinsamen Startlinie gelangt usw. Auch die Gewinne sind keine wirklichen: Nach je einem Treffer kann man eine Linie ziehen, was irgendeinen Vermögensgegenstand bedeutet, in anderen Spielen macht man Geländegewinne usw., und zum Schluß ist der mit dem größten Grundstück oder mit dem meisten Vermögen der Sieger. In manchen Fällen bestraft gerade der Erste den Letzten, obwohl dies eher die Aufgabe der kleinen Gemeinschaft ist. Bei jenen Spieltypen, bei denen außer dem Messer keine anderen Hilfsmittel benutzt werden, muß in zwei Fällen der Letzte das ausgeschnittene und sorgfältig wieder eingepaßte Bodenstück finden - gelingt es ihm, darf er es dem übrigen hinzuschneiden, bei einer Variante packt irgendwer dem Letzten den ausgeschnittenen Grassoden auf den Rücken und läßt ihn diesen auf allen Vieren schleppen. Wo dagegen auch Pflöcke benutzt werden, muß der Sucher die versteckten Holzstücke finden oder den in den Boden getriebenen Stock mit den Zähnen herausziehen. Die Verlierer werden zudem noch geneckt und verspottet.

Unter den etwa 40 Spielvarianten gibt es kaum zwei völlig gleiche, deswegen kam es zu der übereilten Feststellung, daß den Taschenmesserspielen fast nur das Messer gemeinsam sei. Die Typologisierung ist tatsächlich nicht leicht, aber keinesfalls undurchführbar; auf jeden Fall erschweren einige gemischte oder unvollständige Varianten die Situation, des weiteren die Überdeckungen bei unterschiedlichen Spielen (z. B. die Wurfarten, die Bestrafung der Letzten usw.). Bisher gelang es, 9 Spieltypen zu unterscheiden, deren Varianten sich mindestens so unverhältnismäßig verteilen wie die landschaftlichen Vorkommen: Die 3 wichtigsten Typen ha- 
ben 30, die übrigen 6 insgesamt 10 Varianten. All das ist nicht nur ein Beliebtheitsindex, sondern verweist auch auf die Einseitigkeit der Sammler. So ist es zweckmäßig, die einzelnen Typen und Typenfamilien ihrer Häufigkeit und Kompliziertheit gemäß darzustellen.

1. Kein wirklich unterhaltsames Gesellschaftsspiel, eher ein grober Scherz ist das Erschrecken durch Niederstechen. Es hat keinen besonderen Namen, auch die Sammler erwähnen ihn nicht, Angaben gibt es nur aus der Tiefebene. Jemand sticht überraschend mit dem offenen Taschenmesser nach einem anderen, wobei er sein Messer umdreht und den anderen mit dem Griff berührt, über seinen Schrecken lachend.

2. Die Sammler haben unbegründet eines der virtuosen Geschicklichkeits-Taschenmesserspiele fortgelassen, da es individuellen Demonstrationscharakter hat, vor Zuschauer(n) stattfindet und zum Nachmachen, zum nachahmenden Wettbewerb animiert. Auch dieses Spiel hat keinen besonderen Namen, gewiß war es nicht nur in der Tiefebene bekannt, weil es beispielsweise die Jungen in der Hauptstadt bis heute mit dem Bleistift spielen! Der Spieler legt seine Innenhandfläche auf, spreizt die Finger, faßt das Messer mit der anderen Hand und sticht in schnellem Tempo und wechselnder Reihenfolge (,übertritt“ beim Daumen beginnend, dann vom kleinen Finger her einzeln und danach im Doppelschritt die Finger, usw.) in die Freiräume zwischen den Fingern. Bei diesem Spiel geht es „nur“ um die Geschwindigkeit und darum, das alle Finger heil bleiben. Wenn überhaupt ein Wettbewerb stattfindet, siegt derjenige, der der Schnellste ist und am abwechslungsreichsten zusticht.

3. Das unter dem Namen Mit der Spanne Messen bekannte Taschenmesserspiel aus dem Kleinen Tiefland ist vermutlich sekundär, durch den Wechsel des Spielgerätes aus einem anderen Wurfspiel entstanden. Da es ein Wettkampf ist, spielt man mindestens zu zweit. Das halbgeöffnete Taschenmesser wird an der Startlinie mit der Spitze in den Boden gestochen, am Griff fassend so in die Luft geworfen, daß es wirbelt und so weit entfernt wie möglich auf den Boden trifft. Der Wurf gilt, wenn die Spitze des Messers sich so in die Erde bohrt wie vor dem Wurf. Die Spieler werfen abwechselnd und entfernen sich mit jedem gültigen Wurf weiter von der Start-linie. Sieger ist, wer am weitesten kam. (Die Entfernung wird im übrigen mit der Spanne gemessen, woher das Spiel seinen Namen bekam.)

4. Der Zielwurf mit dem Taschenmesser wird nur aus der Tiefebene erwähnt, war aber gewiß auch anderswo bekannt. Man konnte ihn zwar auch allein spielen, aber zum richtigen spielerischen Wettkampf wurde er nur durch Beteiligung von zwei oder drei Mitspielern. Aus einigen Metern Entfernung nahm man einen Bretterzaun oder Baumstamm zum Ziel, Bedingung aber war nicht der genaue, sondern der gültige Wurf, das Messer mußte im Holz steckenbleiben. Bei einer Variante warf man fünfmal und zählte die gültigen Würfe, bei einer anderen Variante spielte die Wurfzahl keine Rolle. Das war auch davon abhängig, wie geworfen wurde: Beim Fünfertreffer wurde das offene Messer an der Spitze gefaßt geworfen, natürlich mußte es sich dann, um ins Ziel zu treffen, drehen oder wirbeln. Das Werfen bei der weniger festgelegten Variante war vielleicht etwas leichter: Das offene Messer wur- 
de mit der aufs Ziel gerichteten Spitze auf die flache Hand gelegt und mußte mit kräftigem Schwung ins Holz getrieben werden. Es gab weder Belohnung noch Strafe, der Geschickteste musste sich mit dem Erfolgserlebnis begnügen.

5. Bei den drei Varianten eines Taschenmesserspiels aus der Tiefebene wird der Gegner durch Würfe zu immer stärker gespreizte Fußstellung gezwungen, bis er aufgibt. Daher stammt auch die Benennung Spanner oder Sprenger. Es erinnert etwas an Typ 4, ist aber komplizierter. Die Varianten selbst unterscheiden sich teils durch die Wurfart und teils durch die Anzahl der Spieler, letztere bestimmt auch die Form der Spielfläche. Bei der einfacheren Zweiervariante 5a stehen die Gegner in Hab-achtStellung einige Meter voneinander entfernt einander gegenüber. Wer beginnt, wird ausgelost, die Wurfart ist nicht festgelegt, wohl aber, daß sich das Messer einen halben oder höchstens einen Meter von dem Gegner in die Erde bohren muß, woraufhin der Gegner den Fuß, dem das Messer am nächsten ist, um diese Entfernung seitwärts setzen und in dieser nachteiligeren Stellung werfen muß. Wer die immer größere Spreizstellung nicht mehr aushält, gibt das Spiel auf. Üblicherweise ist der Kampf aber nicht so bald beendet, weil sich die Gegner bemühen, ihr Messer zwischen die gespreizten Beine des Gegners zu werfen; glückt es, können sie wieder die Habacht-Stellung einnehmen und das Spiel in günstigerer Lage von neuem beginnen. Bei der Variante 5b mit mehreren Spielern stellen sich diese entlang eines Kreises von mehreren Metern Durchmesser gegnüber auf. Die Regeln stimmen mit Variante 5a überein, doch wird das Messer nicht mit der Spitze, sondern dem Griff nach vorne, also in umgekehrter Richtung geworfen, so daß es sich in der Luft drehen muß. Wenn es schräg in den Boden fährt, aber zwei Finger noch darunter geschoben werden können, ist der Wurf gültig. Bei diesem Kreisspiel bekommt der Sieger für jeden herausgeworfenen Spieler ein Holzstück, also einen „Knochen“ Sieger ist, wer die meisten Knochen hat. Bei Variante 5c werfen die Spieler das an der Spitze gefaßte Messer, daß in der Luft herumwirbeln muß; verboten war, mit zwei großen Würfen den Gegner hinauszudrängen, und wer den zu breiten Spreizschritt nicht mehr aushielt, konnte ohne „Schande“ aufhören, wenn er sein Messer zwischen die Füße seines Gegners plazierte. (Die Beschreibungen erwähnen es zwar nicht, doch bildeten die Varianten a, b und c einst vermutlich ein einheitliches Ganzes.)

6. Das Suchenlassen ausgeschnittener und wieder eingepaßter Rasensoden durch einen oder mehrere Sucher sind vermutlich Abschlüsse komplizierterer Taschenmesserspiele, die sich mit der Zeit verselbständigt haben. Über diesen Typ gibt es nur zwei Angaben aus dem Kleinen Tiefland. Nach Variante 6a schickt die einige Mann starke Gruppe einen durch Auslosung als Sucher fort, sein Name ist Stier. (Es stammt vielleicht daher, daß er die Rasensoden mit seinem Taschenmesser so stößt, wie der Stier seine Hörner benutzt.) Die übrigen kratzen in eine größere Rasenfläche einen Kreis, innerhalb dessen jeder einen faustgroßen Rasensoden aussticht, aber sogleich wieder sorgfältig einpaßt. Der zurückgerufene Stier kommt brüllend an und versucht, mit der Spitze seines Messers die Grassoden zu bewegen. Ist er ein guter Beobachter, findet er schnell einen, hebt ihn mit der Messerspitze heraus und wirft ihn hinter den Weglaufenden her. Wen er trifft, mit dem tauscht er den Platz. 


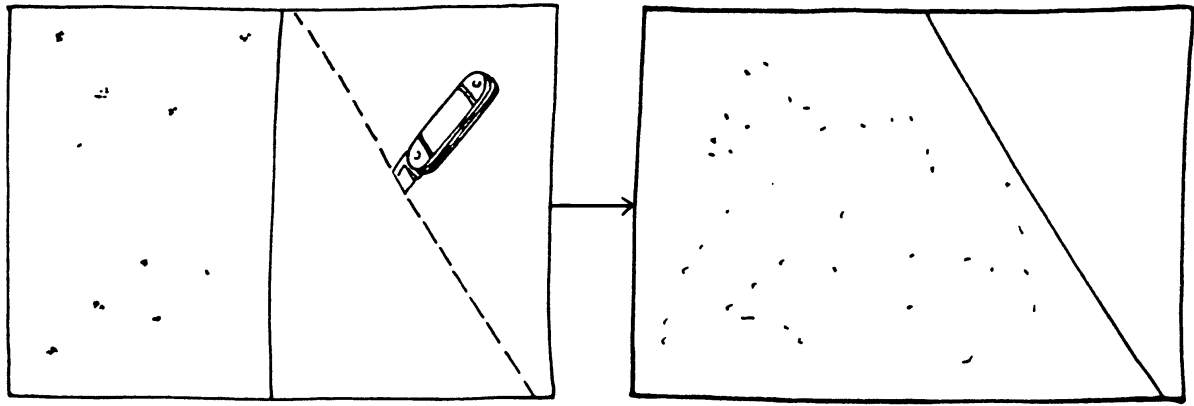

Abb. 2. Gebietserwerb gemäß dem Einfallswinkel des Taschenmessers (HINTALAN-LÁZÁR 1980: 156-7)

Variante 5b ist ein Gruppenspiel zweier Paare. Nach der erwähnten Auslosung mittels Spucken geht das eine Paar als Sucher weg, die beiden anderen Spieler schneiden je einen keilförmigen Grassoden aus und passen ihn wieder genau ein. In beliebiger Entfernung, mindestens aber einige Spannen weit, sticht jeder sein Messer so vor seinen Keil, das die Schneide auf die Spitze des Keils weist. (Somit ist die Richtung gegeben, die Entfernung aber muß herausgefunden werden.) Man ruft die Sucher zurück und fragt sie: Wie viele Spannen von der Messerschneide? Die Sucher beginnen beide gleichzeitig mit der Suche, den glücklich gefundenen Grassoden werfen sie auf die Weglaufenden, damit sie im nächsten Durchgang mit ihnen tauschen können. (Spielbeendigung durch Bewerfen begegnen wir auch bei den folgenden Spieltypen.)

7. Von dieser Typenfamilie sind acht Varianten aus drei größeren Landschaften bekannt, was auf eine erhebliche Verbreitung und Beliebtheit verweist. Sie unterscheidet sich von Typ 6 mehr als vom Typ 8, ist aber trotz gewisser Ähnlichkeiten mit letzterem mit Sicherheit als selbständiger Spieltyp zu betrachten. Sie ist in drei Untertypen zu gliedern, die jeweils weitere Varianten besitzen: Die vier Varianten des Typs $7 \mathrm{a}$ wurden in der Tiefebene und im Kleinen Tiefland sowie in Transdanubien, je eine Variante von Typ 7b und 7c in der Tiefebene und im Kleinen Tiefland aufgezeichnet. Gemeinsam sind ihnen der virtuelle Vermögenserwerb von beweglicher oder unbeweglicher Habe, der Gewinn der Sieger und die Bestrafung der Verlierer.

Der relativ einfachere und einheitlichere Untertyp 7a wurde von 2-4-6 Teilnehmern gespielt. Auf ebener Fläche wurde ein Quadrat, Rechteck oder Kreis in der Spielerzahl entsprechende gleiche Teile geteilt; waren diese groß genug, konnte man sich in seinen auch hineinstellen. Man war bemüht, durch gültige Würfe Raum $\mathrm{zu}$ erobern, das bringt auch die Benennung Landesstückelung-Landmachung zum Ausdruck. (Abb. 2) Im Falle von 4-6 Spielern konnten sich Paare zusammentun, die ihre gegenseitigen Gebiete nicht angriffen. Man begann mittels Auslosung und folgte dann dem Uhrzeigersinn. Die Wurfart war nicht immer festgelegt. Das Messer wurde in das Gebiet des Nachbarn oder Gegenüberstehenden geworfen, und dem Winkel des Einstiches entsprechend schnitt man nach vorn und hinten bis zur 
Grenze, das sich ergebende Stück unter Beseitigen der früheren Grenze dem eigenen Gebiet zuschlagend. Fiel das Messer nicht in ein Nachbar-, sondern in ein entfernteres Feld, wurde dies als „Kolonie“ in Reserve gehalten, im Falle eines gegenüberliegenden Kreissegments kratzte man den Anfangsbuchstaben seines Namens hinein. (In beiden Fällen rechnete man damit, im Laufe der Gebietsgewinnung bis dahin zu kommen und es in das eigene vergrößerte Land einzubeziehen. Im Falle des Kreissegments konnte man dem momentanen Verlierer anbieten, das größere Stück zu behalten.) In der einen Tiefebenenvariante wurde die Art der aufeinanderfolgenden Würfe ebenso bestimmt wie beim Typ 9 (s. dort). Wer sein eigenes Gebiet verloren hatte, schied aus; Sieger war, wer das ganze Gebiet gewonnen hatte, eventuell erhielt er auch eine Belohnung.

Charakteristisch für Untertyp 7b war nicht der Gebietsgewinn, sondern der Vermögenserwerb, dementsprechend schrieb man in seine eigene Rubrik nach erfolgreichem Wurf irgendein vereinbartes Zeichen hinein. Die Variante Katzenspiel aus der Kleinen Tiefebene war die einfachere; das geworfene Messer konnte fast fallen, wie es wollte, es wurde gepunktet: bohrte es sich mit der Spitze in den Boden, 100; fiel es mit der Schneide nach unten, 60; auf den Rücken, 50; fiel es mit der gekerbten Seite nach oben hin, 10 Punkte. Sieger war, wer die meisten Punkte hatte. Die Tiefebenenvariante war komplizierter: Das halb geöffnete Messer wurde in den Boden gesteckt und am Griff hochgerissen. Fiel es flach auf den Boden, gewann der Werfer momentan nichts, ansonsten hing der Gewinn vom Einstichwinkel ab: Bohrte sich die Messerspitze in die Erde und berührte auch der Griff den Boden, d. h. war das Messer in Ausgansstellung, war der Gewinn ein Pferd (dessen Anfangsbuchstabe, ung. L, wurde in die eigene Rubrik eingeschnitten); gelang der Einstich etwas schief, war der Gewinn „nur“ eine Kuh (T); fiel das Messer auf den Rücken, lag der Griff also auf dem Boden und die Klinge stand gerade nach oben, dann war der Gewinn ein Schloss (K, auch das Spiel selbst hieß Schloßmachen); fiel es genauso, aber im schrägen Winkel und fiel nicht um, war die Belohnung ein Palast (P); bohrte sich das Messer zwar in den Boden, aber der Griff hing in der Luft, dann maß man die Lücke mit dem Finger, und der Gewinn war so viel Joch Feld, wie viele Finger dazwischen paßten (2-3 F); man verlor alles, wenn das Messer auf das Gelenk fiel, also Griff und Klinge V-förmig nach oben standen, denn das bedeutete Brunnen, alles war also in den Brunnen gefallen; die bisherigen Gewinne mußten gestrichen werden, doch konnte der Verlierer wieder mit Sammeln beginnen. Es konnten umstrittene Messerstellungen vorkommen, die nannte man als Priester stehen und ließ sie unbewertet. Sieger war der mit dem meisten Vermögen, in erster Linie zählte die Größe des Grundbesitzes, bzw. sie war bei Streitfällen entscheidend.

Die beiden Varianten von Untertyp 7c hatten keinen Gebietsbesitz oder Vermögenserwerbung zum Ziel, sondern nach gelungenen Würfen mußte aus der Grube des Gegners möglichst viel Boden beschafft werden. In diesem Fall war die aus dem Kleinen Tiefland die einfachere, und wie viele auch immer spielten, sie gehen im Grunde in Paaren vor. Jeder hebt vor sich im Rasen eine kleine Grube aus. Der ausgeloste Beginner fragt seinen Partner, ob er die glatte oder eingekerbte Seite der Klinge wählt. Er wirft das Messer sehr hoch, damit es gut wirbelt, es muß sich aber 
nicht in die Erde bohren, sondern soll möglichst flach hinfallen. Hat der Gefragte die obenliegende Seite erraten, kann er aus der Grube des Werfers ein so großes Stück herausschneiden, womit er fertig werden muß, während ein zweizeiliger Spruch von zweimal sieben Silben aufgesagt wird. Dieses Bodenstück heißt Fett, im Laufe des Spiels versucht man dies mittels gültigen Würfen zu vergrößern. Wenn der Gefragte die richtige Messerseite nicht erraten hat, kann der Werfer ein Stück aus dessen Grube herausschneiden. So wechseln die Paare einander bis zum Ende $a b$, weil die gelungenen Antworten den folgenden Frager bzw. Werfer ergeben. Sieger ist, wer den größten Haufen Boden, also Fett, hat. Die Tiefebenenvariante ist nicht nur komplizierter als diese, sondern bereits ein richtiges Theaterstück. In der Mitte des bezeichneten Spielkreises wird eine größere Grube gegraben, sie ist die des fiktiven Königs. In Entfernung je einer Spanne von dieser gräbt sich jeder seine kleinere Grube, das Spiel folgt dann dem Uhrzeigersinn. Bei dieser Spielvariante kann ebenfalls die glatte oder gekerbte Klingenseite gewählt werden; die letztere ist kóka, das Spielt heißt kókázás (etwa Kóka spielen) (Um der Chancengleichheit willen spielt man nur mit einem Messer.) Der ausgeloste erste Spieler versteckt das Messer in seinen zusammengelegten Handflächen, der folgende muß wählen, ohne es zu sehen, oder auf einen der Handrücken schlagen. Hat er beispielsweise auf die glatte Klingenseite geschlagen, ist sein Wurf dann gültig, wenn das an der Klinge hochgeworfene und wirbelnd herunterfallende Messer mit der glatten Klingenseite nach oben zu liegen kommt. Andernfalls gewinnt er nicht und der Nächstfolgende kommt dran. Während das Messer in der Luft wirbelt, versuchen die Werfer, das glückliche Fallen zu „fördern“, im beschriebenen Fall mit mit dem Text: kóka, dreh dich, fall' auf die glatte Seite! Der erfolgreiche Werfer könnte aus der Grube jedes beliebigen Mitspielers ein Stück herausschneiden, doch haben die Freunde meist verabredet, einander nicht zu schaden, ja es kommen auch Verschwörungen vor, daß alle die Grube desselben Mitspielers erweitern, damit das Spiel schneller beendet wird. Der glückliche Werfer macht sich in den meisten Fällen nicht sogleich über die ausgesuchte Grube her, sondern erschreckt vorher die Eigentümer der Gruben: er hebt eine Messerspitze Erde an die Lippen, sagt - o, wie bitter! - und läßt die Grube in Ruhe, um dann - o, wie süß! - zu rufen, aber wieder weiterzusuchen, bis er schließlich - das ist die süßeste! - ruft und diese plündert. Diese Prozedur ist streng geregelt und auch nicht allzu leicht: Der Spieler darf nur mit einer Hand arbeiten, schneidet mit der Messerspitze ein flaches viereckiges oder rundes Stück aus, hebt es mit dem Messer heraus, legt es sich auf die Handfläche, legt sein Taschenmesser darauf, schüttelt es ein wenig, und wenn nur eine Krume davon herunterfällt, muß er das kleine Rasenstück an seinen Platz zurücktun; ansonsten kann er es neben seine eigene Grube setzen. Wenn der Rand der erweiterten Grube eines der unglücklicheren Spieler die mittlere, die des Königs, erreicht, wird mit dem Bodenerwerb aufgehört. Die Königsgrube wird gemeinsam eingeebnet, danach tut das jeder mit seiner eigenen aus der erworbenen Erde, die Freunde helfen sich gegenseitig, eventuell hilft man auch den Pechvögeln mit übriggebliebener Erde aus; aus wessen Grube aber dann immer noch Erde fehlt bzw. wo die Grube noch am größten ist, der wird nach gemeinsamer Einebnung der Fläche bestraft, er muß die sog. Kókascheiße 
wegschaffen. Man verbindet ihm die Augen, drückt ihm eine übriggebliebene Erdscholle in die Hand und führt ihn so lange herum, bis er die Orientierung verloren hat. Dann lassen sie ihn die Scholle wegwerfen, führen ihn zurück, nehmen ihm die Binde ab und lassen ihn den Erdklumpen suchen. Unterdessen vesuchen sie ihn zu beirren und hänseln ihn. Findet er die Scholle trotzdem, darf er sie nach irgendjemandem werfen, und dieser wirft sie zurück. Manchmal werfen sie einander mit dem Brocken so lange, bis er völlig zerfällt.

8. Einige Momente dieses Typs sind zwar mit denen von Typ 7 und 9 verwandt, er ist aber in vollem Maße als selbständig zu betrachten. Es ist ein Geschicklichkeitswettbewerb, bei dem man vom Rande eines Kreises mittels einer geglückten Wurfserie die Mitte erreichen muß; hier fungiert das Taschenmesser nicht nur als Wurf-, sondern auch als Meßgerät, und die erzielte Entfernung wird üblicherweise mit Pflöcken gekennzeichnet. Seine zehn Varianten weisen auf seine beachtliche Beliebtheit hin, die Verteilung auf die einzelnen Landschaften ist gleichmäßig: Tiefebene (3), Siebenbürgen (3), Transdanubien (2), Kleines Tiefland (2); Untertypen gibt es keine, je mehr man sich aber von der Tiefebene entfernt und sich der Gegenwart nähert, vereinfacht sich auch das Spiel etwas.

Die transdanubischen Varianten werden von 3-4 Jungen gespielt. Sie kennzeichnen die Mitte des Kreises, indem sie einen runden Grassoden ausschneiden und umgekehrt wieder einsetzen oder einen größeren Pflock als Pfaffe einschlagen. In einer Entfernung von zwei Spannen oder Messerlängen stecken sie ihre kleineren, Ministranten genannten Pflöcke in den Boden. Der eine eines bestimmten Paares kann unter den beiden Klingenseiten wählen, wie bei anderen Typen schon erwähnt wurde, sein Partner wirft das Messer hoch, und wenn es mit der gewählten Seite nach oben hinfällt, darf der glückliche Gewinner als erster werfen. Jetzt aber muß er das Messer nicht auf die Seite fallen lassen, sondern zwischen zwei Fingern gefaßt mit voller Kraft auf den Boden werfen, daß es möglichst tief hineinfährt, weil er der Tiefe entsprechend ihr Stöckchen so viel weiter zur Mitte hin verrücken kann. Bei der einen Variante können erfolgreiche Würfe hintereinander wiederholt werden, die Spieler lösen einander nur nach Fehlwürfen ab. Dem Letzten drücken sie den Priester in die Hand und führen ihn mit verbundenen Augen, während sie ihn ängstigen: - $\mathrm{Pa} ß$ auf, hier ist der Brunnen! Fall nicht hinein! - Wenn er sein Orientierungsvermögen schon genug verloren hat, lassen sie ihn den Pflock wegwerfen, führen ihn an die Ausgangsstelle zurück, nehmen ihm die Binde ab, und er muß den Stock suchen. Seine Gefährten „helfen“ ihm: - kalt, warm! -; den gefundenen Pflock darf er auf die Spötter werfen, und einer von ihnen wirft ihn zurück.

Auch die Varianten des Kleinen Tieflandes werden zu viert gespielt, und der zentrale Pflock heißt bei der einen ebenfalls Pfaffe, bei der anderen Stier. In einer Entfernung von 3-4 Spannen vom Mittelpunkt steckt man bei diesen Varianten je zwei Pflöcke nebeneinander in die Erde, die kleinen Stiere; den größeren von etwa 15 $\mathrm{cm}$ außen, den kleineren von $8 \mathrm{~cm}$ innen; der Kreisumfang kann sogar 2-3 m betragen (Abb. 3). Hier ist die Aufgabe gegenüber der transdanubischen Variante vierfach, weil der Weg hin und zurück mit beiden Pflöcken gemacht werden muß. Die Wurfarten sind mit denen der vorigen identisch, aber die Bestrafung des Letzten 


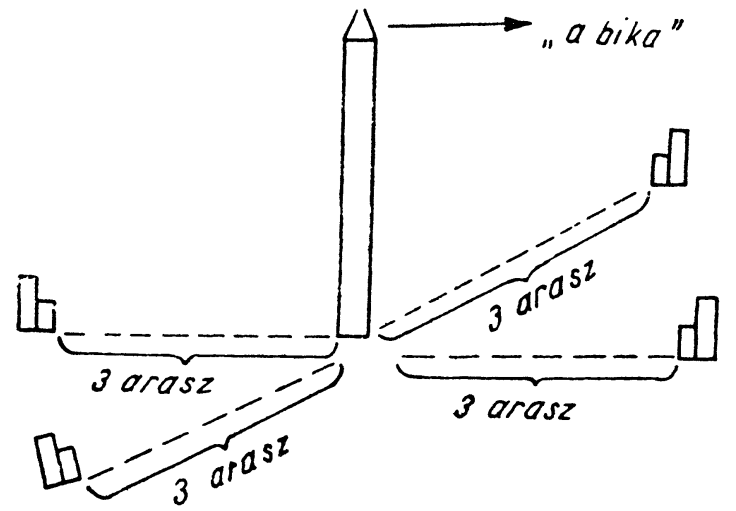

Abb. 3. Stellung des zentralen größeren Pflockes und der individuellen kleineren Pflöcke (BAKOS 1953: 263)

weicht ab: entweder muß er den tiefer eingeschlagenen zentralen Pflock mit den Zähnen herausziehen oder die von ihm nach bekannter Weise mit verbundenen Augen weggeworfenen sämtlichen Pflöcke zusammensuchen; unterdessen wird auch er ermuntert: - Himmel, Meer, Wasser, Feuer!...

An den Varianten aus der Tiefebene können 4-6, eventuell sogar 8-10 Spieler teilnehmen. In die Mitte eines Kreises mit etwa $2 \mathrm{~m}$ Durchmesser schlägt man einen etwa 40-50 cm hohen Pfahl ein, in einzelnen Varianten nennen auch sie ihn Stier, in anderen Fällen ist dies der Name des Gewinns. Die Länge des eigenen Stockes (einer oder zwei) ist etwa $10 \mathrm{~cm}$; wenn sie nicht in den Boden gesteckt, sondern in der Hand gehalten werden, wird sitzend geworfen. Die Reihenfolge und Wurfarten entsprechen den vorangegangenen Varianten, auch die Vorrückentfernung wird auf ähnliche Weise gemessen, dagegen muß der Mittelpfahl nur erreicht werden, ebenso wie bei der transdanubischen Variante, und die Läufe können wiederholt werden. Jedes Erreichen des Ziels ist ein Stück Großvieh wert (Pferd oder Stier), und Sieger ist, wer am meisten Tiere besitzt. Der Verlierer wird weit fortgeschickt, währenddessen versteckt jeder seine eigenen Pflöcke im Boden, und der Sucher muß sie unter Ermunterungen wie - Wasser-Feuer oder warm-kalt - mit der Messerspitze in den Boden stechend finden; wessen Pflock er gefunden hat, der wird fortgeschickt; so wird die Suche abwechselnd fortgesetzt, bis alle Pflöcke gefunden sind.

Eine der siebenbürgischen Varianten wird Pfaffe genannt, die andere Messerwerfen; der in die Kreismitte gesteckte Pflock von etwa $30 \mathrm{~cm}$ heißt: Dorfstier; das eigene kleinere (spannen- oder fingerlange) Pflöckchen hat keinen Namen, es wird in Entfernung von etwa 3 Messerlängen vom Mittelpunkt eingesteckt. Es kann auch sitzend gespielt werden, dann aber wird das Messer nicht so tief wie möglich in den Boden geschleudert, sondern die Spieler werden sich darum bemühen, es auf die gewählte Klingenseite zu werfen. Dementsprechend wird gemessen: Liegt die gekerbte Klingenseite oben, kann man sich dem Mittelpunkt um eine ganze Messerlänge nähern, im Falle der glatten Seite muß man eine Runde aussetzen. Bei ande- 
ren Varianten wird das Messer in die Erde getrieben und die Tiefe als Maß des Vorrückens genommen. Es kommen auch gemischte Varianten vor, beispielsweise hilft das in der Erde steckende Messer dem Werfer eine halbe Messerlänge voran, während die obenliegende gekerbte Klingenseite eine ganze Messerlänge bedeutet. Wer Letzter geblieben ist, wird der Sucher. Der Sieger ritzt um den Mittelpfahl einen kleineren Kreis ein, innerhalb dessen er seinen eigenen kleineren Pflock ganz in die Erde steckt, welchen der Sucher mit der Messerspitze finden muß, aber nur zehnmal in die Erde stechen darf. Wenn er ihn gefunden hat, muß der Sieger Sucher sein. Nach einer anderen Bestrafungsart läßt man den Sucher mit verbundenen Augen irgendwo ein Steinchen oder einen Kiesel wegwerfen, den er dann ohne Binde finden muß. Gelingt es ihm nicht, wird er spielerisch verprügelt.

Die siebenbürgischen Varianten kennzeichnet eine totale Mischung, denn in der ersten Runde wird mit halboffenem und in der zweiten auch mit offenem Messer geworfen, in beiden Fällen muß aber die Spitze im Boden stecken, weil an seiner Tiefe die Vorrückweite gemessen wird; auch das Meßwerkzeug ist ein anderes dem wir allerdings in anderen Landschaften schon begegnet sind -, man nimmt nicht das Messer, sondern das Pflöckchen. Wer den Mittelpunkt als Erster erreicht, hat gewonnen; über die Bestrafung des Letzten gibt es allerdings keine Angaben.

9. Es ist wohl kein Zufall, daß dieser vielnamige Spieltyp der schwierigste, komplizierteste und auch beliebteste ist, des weiteren ist er auch durch den meisten gemeinsamen Zügen mit den anderen verbunden. Seine 12 Varianten haben $9 \mathrm{Be}-$ nennungen, seine 6-12 verschiedenen Griff- und Wurfarten haben gerade 30 verschiedene Namen. Die Verteilung nach Landschaften ist bei ihm am ungleichmäBigsten: Tiefebene (7), Kleines Tiefland (2), Transdanubien (2) und Siebenbürgen (1), dennoch ist er als selbständiger Typ zu betrachten. In seinem Wesen ist er ein in immer schwieriger werdender Reihenfolge und mit ausgewählten Griff- und Wurfarten stattfindender Geschicklichkeitswettbewerb; all dies läßt ihm zum typischen Taschenmesserspiel werden. (Wer es besteht, kann tatsächlich alles!) Es ist das Lieblingsspiel vor allem der 8-14jährigen Jungen. Ungeachtet seiner Schwierigkeiten wird gerade dies von den größten Gruppen gespielt, die Ausnahme sind 2-4-6, üblich dagegen 8-10-12-14 Spieler, besonders beliebt ist es bei Kindern im Schulalter. Die Spielfläche kann abhängig von der Spielerzahl ein hand- oder $10 \times 10 \mathrm{~cm}$ großer, aber ebenso auch ein Kreis mit $2 \mathrm{~m}$ Durchmesser sein. Sie wird gründlich gesäubert, nach einer Tiefebenenangabe wird sie kreuzweise eingeschnitten und abgetragen; der geglättete Boden wird auch leicht festgestampft. Da der Wettkampf keine Bewegung im Raum verlangt, spielt man auf einer Stelle stehend, bei weniger Mitspielern kann aber auch im Kreis sitzend gespielt werden. Wir kennen auch eine Variante, bei der zuerst kniend und dann stehend geworfen wurde.

Die Auslosung geschieht auf die schon erwähnte Weise mittels Hochwerfen und seitlichem Fallen des Messers mit Hilfe der richtig gewählten Klingenseite. Im weiteren $\mathrm{mu} ß$ in zunehmend schwieriger werdender Reihenfolge das Messer angefaßt und geworfen werden, aber aus welcher Lage auch immer, muß es mit der Spitze im Boden stecken. Steckt das Messer schief im Boden, muß zwischen Messer und Boden zumindest der kleine Finger, nach strengeren Regeln zwei Finger passen, damit 

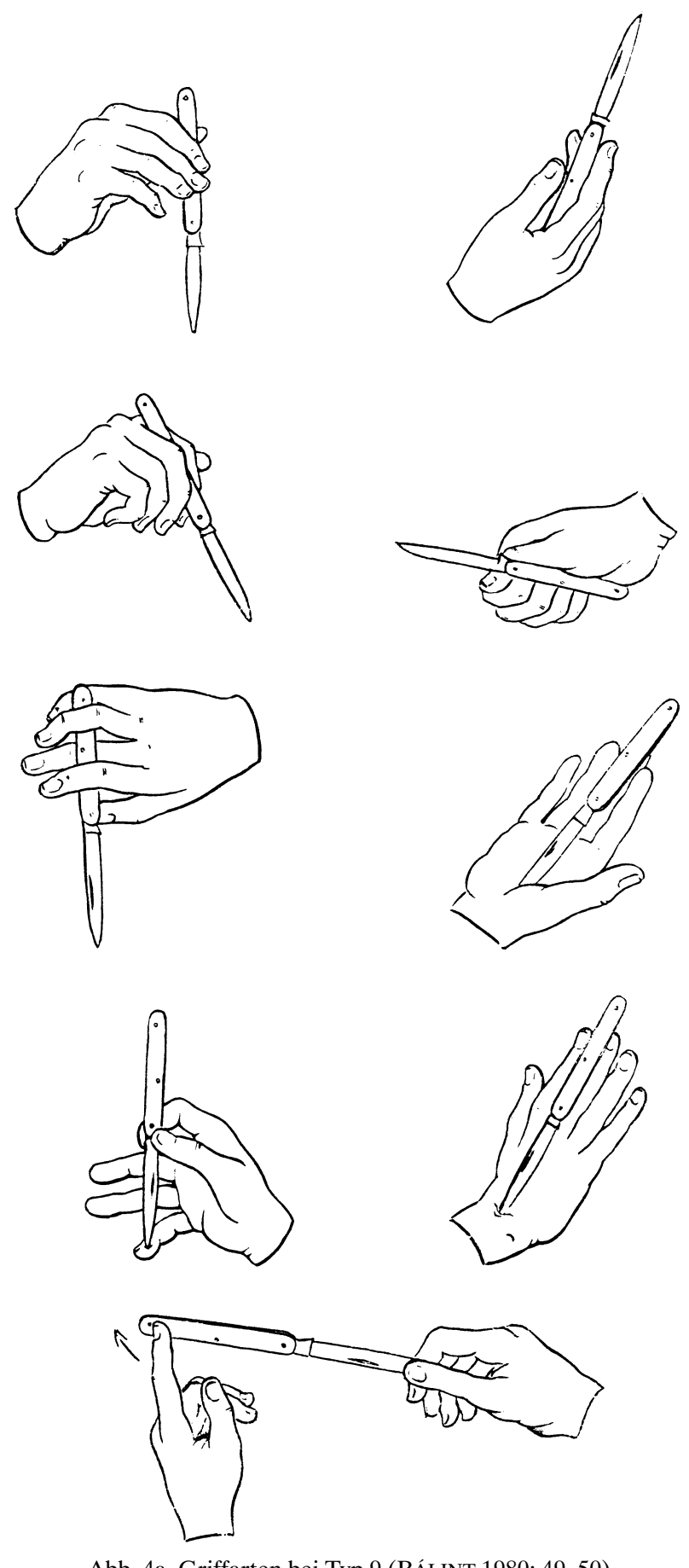

Abb. 4a. Griffarten bei Typ 9 (BÁLINT 1980: 49-50) 

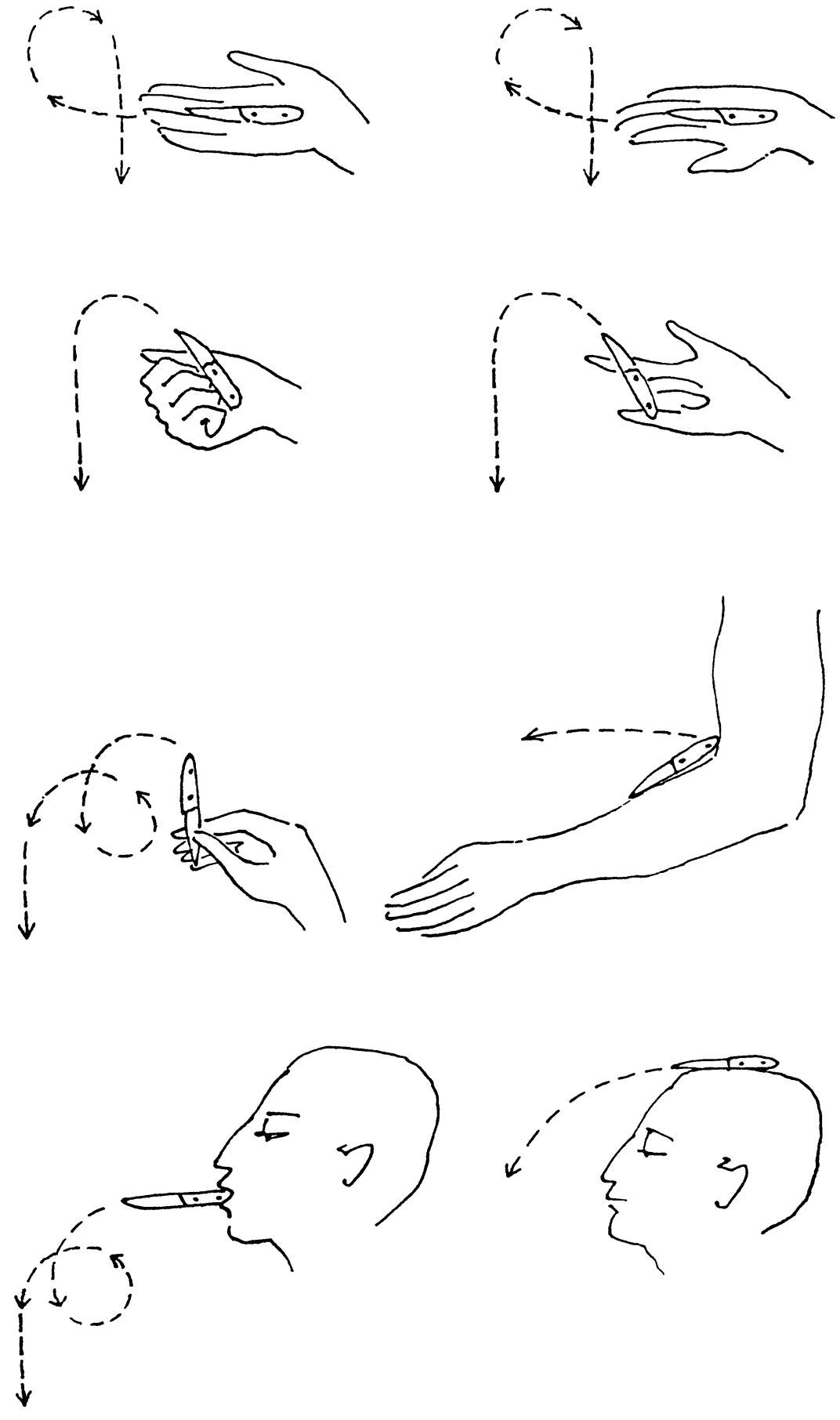

Abb. 4b. Wurfarten bei Typ 9 (SALÁNKI o. J. Beilage) 
der Wurf gilt. Einer einzigen Angabe aus dem Kleinen Tiefland gemäß mußte beim 9. Wurf der Messergriff in der Erde stecken, was allerdings fast unmöglich zu schaffen war. Eigentlich war es nicht schwer zu erreichen, daß das Messer in den Boden eindrang, vielmehr waren die Arten des Werfens sehr schwierig: die Griffe und Stellungen. Die 6-12 verschiedenen Varianten lassen sich schriftlich nur schwer und umständlich erklären, sie sind viel einfacher im Bild zu demonstrieren. Während die neun verschiedenen Typennamen bloß spielerische Wortbildungen sind und sich nur seltener deuten lassen (z. B. Taschenmesserwurf und Pfählen in der Tiefebene, Grashüpfer in Transdanubien), sind die 30 Griff- bzw. Wurfartenbenennungen etwas leichter erklärbar: Faust, Handfläche, hohle Hand, Finger, Nagel (je 6 Angaben), Handwurzel, Handrücken (je 5), Mund, Zahn (je 4), Arm, Ellenbogen (je 3), Kinn, Nase, Stirn, Kopf, Schulter (je 1), die übrigen Benennungen können sich auf das Taschenmesser, auf den Bogen der Wurfbahn (drehend, spitz, bogig) und auf das Auftreffen (hineinschneiden, aufschlagen) beziehen, und natürlich sind auch dort die unauflösbaren spielerischen Wortbildungen häufig (11). Diese komplizierten Benennungen werden schneller in Vergessenheit geraten sein als die Wurfarten selbst, und es kommt auch vor, daß sich in der Reihenfolge der Terminus wiederholt, obwohl sich die Wurfart ändert, oder auch umgekehrt. All dies ist auf die Rechnung des Vergessens, der Abnutzung oder des Sammelns zu schreiben. Im Grunde folgten den leichteren Griff- und Wurfarten mit den Fingern die schwereren, z. B. vom Mund, Nasenrücken, der Stirn und der Schulter aus. Am abwechslungsreichsten waren sie in der Tiefebene, wo die schwerer werdende Reihenfolge mit dem Wort Schule oder Klasse verknüpft werden konnte. Wessen Würfe gelangen, der hatte die Schule bestanden, die Pechvögel dagegen waren die Sitzenbleiber (Abb. 4a und b).

Zumeist gab es weder Belohnung noch Strafe, andernfalls wurde ähnlich wie bei den übrigen verwandten Spieltypen vorgegangen. Bei einer Variante aus der Tiefebene beispielsweise bedeutete - im Wiederholungsfall - ein Zug das Pferd, bei fehlerfreier Serie den Stier, und schließlich siegte der „reichste“ Spieler. Die Siebenbürger häuften die aus dem Boden geschnittenen Speckseiten auf (vgl. mit dem schon erwähnten Fett!). Die Betrafungsarten kennen wir bereits: Der Sieger schlägt in Transdanubien ein Holzstück mit so vielen Schlägen mit dem Messer in den Boden, wie viele Würfe dem Verlierer mißlungen waren, und letzterer mußte den Pflock mit den Zähnen aus dem Boden ziehen. Dasselbe gibt es auch in der Tiefebene, doch schlägt in dieser Variante nicht nur der Sieger auf den Pflock, sondern alle Spieler, und diesen muß der Verlierer dann mit den Zähnen herausziehen. Es kommt auch das Verstecken der Pflöcke vor, wie ebenfalls schon erwähnt. Da man bei diesem Spieltyp eigentlich gar keine Pflöcke braucht, ist die Übernahme aus anderen Spielen offensichtlich. Wahrscheinlich ebenfalls "fremd“ ist auch die siebenbürgische Lösung, dem Verlierer die erwähnten Speckseiten auf den Rücken zu packen und von ihm so lange auf allen Vieren schleppen zu lassen, bis sie herabgefallen sind. 


\section{ZUSAMMENFASSUNG}

Die auf der individuellen Geschicklichkeit beruhenden Taschenmesserspiele mit Hilfsmitteln waren bei den ungarischen Schuljungen bis in die nahe Vergangenheit beliebt. Ihre Sammlung wurde recht vernachlässigt. Die 38-42 Varianten von 9 Typen entsprechen keineswegs der Wirklichkeit. Da diese für gefährlich gehaltenen Spiele manchmal verboten wurden, lernten die Spieler voneinander; im übrigen gibt es keine Angaben über Verletzungen. Die Dominanz der Tiefebene läßt sich mit der Offenheit der Landschaft und der Wegstrecke der Übernahme erklären. Die Taschenmesserspiele sind international, aber kaum uralt, und trotz ihres scheinbaren Kampfcharakters gibt es kein einziges militärisches Fachwort (z. B. bedeutet der Kreis niemals Burg) in der ansonsten reichen Spielterminologie; es gibt einige feudalistische Züge (König, Palast, Schloß usw.), ähnlicherweise auch einige kirchlicher und schulischer Herkunft (Priester, Ministrant, Schule, Klasse usw.), später sickerte auch die große Politik ein (Land, Kolonie), die Mehrheit der Termini technici blieb aber typisch bäuerlich (Feld, Stier, Pferd, Fett, Speckseite usw.) und stand mit dem virtuellen Vermögensgewinn in Zusammenhang. Mit Ausnahme eines oder zweier Übergangs- und unsicherer Typen sind die Spiele kompliziert und variabel, es gibt keine zwei völlig identische Varianten. Auslosungsart, Reihenfolge der Spieler, Gültigkeit der Würfe und Art der Belohnung und Bestrafung sind in mehreren Typen ähnlich, aber nicht identisch, und die „Handlung“ selbst ist gut unterscheidbar zu typisieren. Dieses Gruppen- (nicht Mannschafts-!) spiel mit Hilfs-mitteln ist auch seines geringen Zubehörs, der einfachen Spielfläche usw. ungeachtet virtuos und geistvoll, es verdient auch weiterhin größere Aufmerksamkeit.

\section{LITERATUR}

BAKOS, József

1953: Mátyusföldi gyermekjátékok (Kinderspiele aus dem Mátyusföld). Új Magyar Népköltési Gyújtemény VII. Hrsg.: ORTUTAY, Gyula.

BÁLINT, Sándor

1957: Szegedi Szótár (Szegediner Wörterbuch), I-II. Budapest.

1980: A szögedi nemzet (Das Szegediner Volk). A szegedi nagytáj népélete, III. Szeged.

GAZDA, Klára

1980: Gyermekvilág Esztelneken. Néprajzi monográfia (Die Kinderwelt von Esztelnek. Eine Volkskundemonographie). Bukarest.

GÖNCZI, Ferenc

1949: Somogyi gyermekjátékok (Kinderspiele in Somogy). Kaposvár.

HAJDÚ, Gyula (Hrsg.)

1971: Magyar népi játékok gyújteménye (Sammlung ungarischer Volksspiele). Budapest.

HINTALAN, László-LÁzÁR, Katalin

1980: Gyermekjátékok. Hévízgyörk, Pest megye (Kinderspiele. Hévízgyörk, Kom. Pest). Szentendre.

KATONA, Imre

1940-44: Csongrádi bicskajátékok (Taschenmesserspiele von Csongrád). Csongrád (Mskr.).

KISS, Áron

1981: Magyar gyermekjáték-gyújtemény (Ungarische Kinderspielsammlung). Budapest (Reprint $\left.1994^{2}\right)$. 
LÁBADINÉ KEDVES, Klára

1984: Barkócakoszorú. Alfalusi (drávaszögi) népi játékok (Elsbeerkranz. Volksspiele von Alfalus [im Drau-Donau-Dreieck]). Eszék.

LAJOS, Árpád

1980: Késjáték (Messerspiel). In: Magyar Néprajzi Lexikon, III: 176-7. Hrsg.: ORTUTAY, Gyula. Budapest.

LÁZÁR, Katalin

1990: Játéktípusok. A játékok rendje (Spieltypen. Die Ordnung der Spiele). In: Magyar Néprajz, VI. Folklór, 2: 544-647. Népzene, néptánc, népi játék. Hrsg.: DÖMÖTÖR, Tekla, Red.: HOPPÁL, Mihály. Budapest.

NovÁK, László

1986: A három város (Die drei Städte). Budapest.

PÉCSINÉ ÁCS, Sarolta

1981: Népi gyermekjátékok Kalocsa környékén (Kinderspiele des Volkes in der Umgebung von Kalocsa). Kalocsa.

SALÁNKI, Szilárd

o. J. Bicskajátékok Kecskeméten és környékén (Taschenmesserspiele in Kecskemét und Umgebung). Jászberény (Mskr.; mit Dank für die Überlassung!) 